\title{
Laparoscopic Inguinal Hernia Repair; Sohag Experience
}

Mahmoud S. Abol Kher, Ahmed Abdel Kahaar Aldardeer,

Osama Abdullah AbdulRaheem, Ayman M. A. Ali, Alaa A. Redwan

Department of Surgery, Faculty of Medicine, Sohag University, Egypt

*Corresponding author: Mahmoud S. Abol Kher, Mobile: (+20) 01010674328, E-Mail: msa201040201040@gmail.com

\begin{abstract}
Background: Laparoscopic inguinal hernia repair is widely adopted and well accepted in many centers.

Objective: We aimed with this study to evaluate the technique regarding its advantages and disadvantages in Sohag University Hospital.
\end{abstract}

Patients and Methods: This was a prospective observational study included 50 patients suffering from inguinal hernia admitted electively to Sohag University Hospital between September 2019 and April 2020. Patients were enrolled for laparoscopic Trans Abdominal Pre Peritoneal (TAPP) repair. Patients were evaluated regarding demographic criteria, clinical picture, operative, and postoperative course after an outpatient follow-up period of 6 months.

Results: The mean operative time in minutes \pm SD was $(125 \pm 12.5)$ for the early 10 patients while it was $(69.8 \pm 24)$ for the last 25 patients. Intraoperative complications were encountered in two cases of visceral injury and one case of bleeding arising from the inferior epigastric artery that was managed laparoscopically without surgical sequelae. The duration of hospital stay ranged from 24-48 hours. Postoperative complications were encountered in 3 patients whom had surgical emphysema which resolved conservatively. Patients returned to full activities in $7.14 \pm 0.96$ days and returned to their work in $14 \pm 2.5$ days. On follow-up, recurrence was not encountered allover the period of 6 months postoperatively.

Conclusion: TAPP repair for inguinal hernias is justified as a feasible, reproducible, and safe technique with rapid recovery and early return to work as well as comparable morbidities to other techniques. It is recommended as the procedure of choice in elective groin hernia repair especially in bilateral cases, with short learning curve requiring little facilities in the center.

Keywords: Inguinal hernia, Laparoscopic surgery, TAPP.

\section{INTRODUCTION}

Inguinal hernia is a prevalent condition worldwide with an incidence of 5\%-7\%. The repair of inguinal hernias is one of the most commonly performed general surgical procedures $^{(\mathbf{1})}$. Approximately 20 million inguinal hernia repairs are performed worldwide every year, which constitutes a significant cost and morbidity burden on the health authorities worldwide. For several decades, the mainstay of treatment was an "open" repair, using a prosthetic mesh with reduced risk of recurrence when compared to an open suture repair ${ }^{(2)}$.

In the early 90s, minimally invasive approaches were introduced. Since then, laparoscopic repair has become increasingly utilised $^{(3)}$. Unlike operations such as cholecystectomy, the laparoscopic technique has not superseded open operations in terms of numbers being undertaken ${ }^{(4)}$. Advocates of the laparoscopic repair describe reduced incision size, reduced postoperative pain and more rapid recovery, with similar complication rates when compared to open repairs ${ }^{(5)}$.

The transabdominal preperitoneal (TAPP) laparoscopic approach also enables intraoperative assessment of the contralateral side for detection and repair of defects missed preoperatively.
Today, laparoscopic TAPP hernioplasty is accepted as a method that is routinely performed in bilateral and recurrent hernia cases as well as in primary unilateral hernia cases $^{(6)}$. Moreover, the posterior approach can be much more reliable for patients with recurrent hernias, in which the mesh will be fixed to the unchanged muscularaponeurotic tissues of the inguinal $\operatorname{area}^{(7)}$. The high cost of the procedure cannot be underestimated but this cost can be offset by lesseasily quantified benefits such as earlier return to work $^{(8)}$.

Opponents of laparoscopic inguinal hernia repair claim that recurrence rates which are a key outcome in hernia surgery are somewhat still encountered, and usually require re-operation ${ }^{(9-}$ 10). and serious intraoperative complications ${ }^{(11)}$ have been found following laparoscopic operations, some of which have been attributed to the early implementation ${ }^{(9)}$ or lack of standardisation of laparoscopic techniques ${ }^{(\mathbf{1 2})}$. These re-operations are also associated with worse outcomes than the primary repair ${ }^{(\mathbf{1 3})}$. Add to this laparoscopic repair demands significant expertise to achieve outcomes comparable with those of open repair ${ }^{(14)}$. 


\section{AIM OF THE WORK}

The purpose of this study was to evaluate laparoscopic TAPP repair in treatment of primary inguinal hernia in Sohag University Hospital.

\section{PATIENTS AND METHODS}

This was a prospective single arm uncontrolled randomized study evaluating outcomes of using TAPP technique; at Sohag University Hospital, Egypt from September 2019 to the end of April 2020 with follow-up till October 2020; follow up period of 6 months on 50 patients admitted electively with a diagnosis of primary inguinal hernia needed surgical treatment.

Only patients who were treated with TAPP repair were included in the study. We excluded patients $<15$ years and > 60 years, patients, recurrent hernias, patients with mega hernias with loss of domain, patients with complicated hernia (irreducible, obstructed, strangulated), patients with abdominal malignancy and cirrhosis or end stage liver disease, patients with history of forced hernia reduction, and pregnant patients or planning to become pregnant.

\section{Ethical considerations:}

This study was performed after approval granted by the Institutional Medical and Ethics Committee of Faculty of Medicine, Sohag University. All participants gave a written informed consent to participate, after receiving an explanation of the study protocol; including benefits and drawbacks of laparoscopic intervention and possible complications. Patients were subjected to complete preoperative clinical evaluation to assess complete surgical fitness. This work has been carried out in accordance with The Code of Ethics of the World Medical Association (Declaration of Helsinki) for studies involving humans.

All surgical procedures were performed under general anesthesia with endotracheal intubation and done by consultant surgeons well experienced in advanced laparoscopy. The patient was placed in supine position with both arms tucked against the side. A 10-mm umbilical trocar was introduced after establishment of pneumoperitoneum, which was maintained at 12$15 \mathrm{~mm}$ of $\mathrm{Hg}$. The patients were then placed in Trendelenburg position with of $10^{\circ}-20^{\circ}$ tilt towards the contralateral side of hernia to expose the inguinal area.

After inspection of the abdominal contents, the important anatomical landmarks and triangles of the inguinal region was identified, A 30 otelescope was used to define the type of hernia and to inspect the inguinal region for any occult hernias (Fig. 1). A 5-mm trocar was then introduced on the right side at the level of the umbilicus, lateral to the rectus muscle. A $10-\mathrm{mm}$ trocar was then inserted at the same level on the left side. A horizontal incision was made in the peritoneum at the most cranial level of the hernia sac (Fig 2). This incision goes from the medial umbilical ligament to the lateral boundary of the internal inguinal ring. First a cranial flap of about $3 \mathrm{~cm}$ was created. A distal flap was then created, exposing the rectus sheath medially, creating a plane in front of the bladder. Distally the dissection was carried down to Cooper's ligament and to the medial side of the femoral vein. Laterally and distally, the dissection involved the offspring of the epigastric vessels and the elements of the spermatic duct that were dissected over at least $3 \mathrm{~cm}$ proximal to the internal inguinal ring. The preperitoneal space was created with monopolar cautery, and blunt dissection (Fig.3). Dissection of the sac with consequent herniotomy is shown in Fig 4. The mesh was then introduced through the $10-\mathrm{mm}$ trocar after it had been folded very much like an umbrella. The mesh, which was heavyweight synthetic non absorbable polypropylene of EGYMESH® of $10 \times 15 \mathrm{~cm}$ size, was unrolled with two graspers and placed in the preperitoneal pocket and made fit for covering the entire dissected area reinforcing the myopectineal orifice (Fig.5).

The mesh was anchored to the Cooper's ligament inferiorly and to rectus muscle superomedial to inferior epigastric vessels using Vicryl® 2-0 (Fig 6).

The peritoneum then was stitched by Vicryl $2 / 0$. Care was taken not to fold the underlying mesh. After the placement of mesh, the peritoneum was closed over mesh with continuous running suture using Vicryl ${ }^{\circledR}$ 2-0 without any gap to prevent adhesions of bowel or omentum (Fig. 7). The trocars were removed, and muscle sheath was closed by Vicryl ${ }^{\circledR}$ zero and skin was closed by Prolene ${ }^{\circledR}$ 3-0.

The main outcome measures were recorded and statistically evaluated (the operative time, intraoperative complications viz; vascular injury, visceral injury, and bladder injury).

Postoperative outcome measures were evaluated and comprised evaluation of surgical complications (postoperative pain, wound infection, wound hematoma, wound seroma, urine retention, testicular pain, testicular swelling), length of hospital stay, off-work vacation, rapidity of return to daily activities and recurrence. The postoperative pain was evaluated by the number of NSAIDS ampoule used during the first 24 postoperative hours.

Oral antibiotic coverage and analgesics were recommended after discharge. 


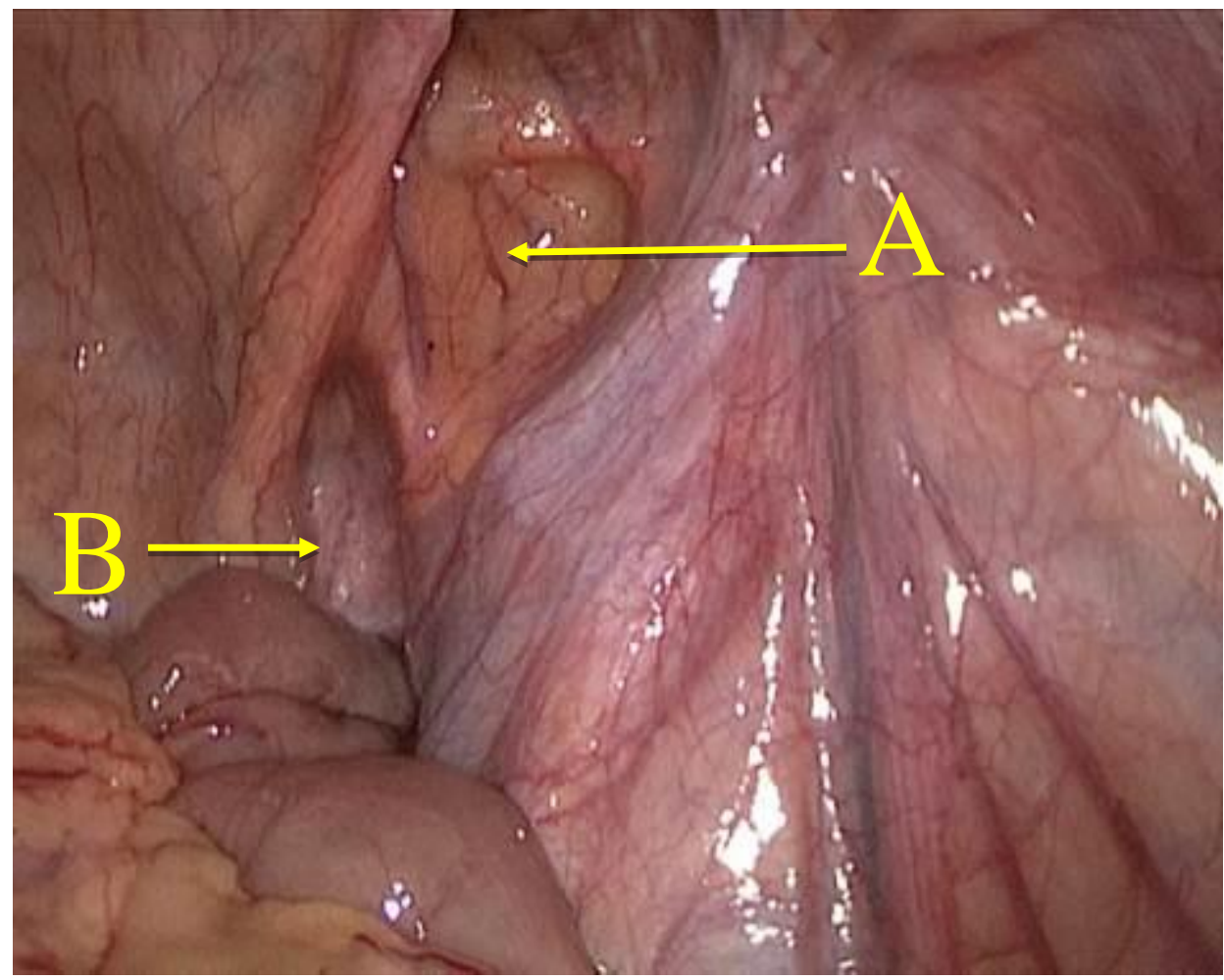

Figure (1): Laparoscopic view of right direct inguinal hernia and femoral hernia. (A: site of direct hernia, B: site of femoral hernia).

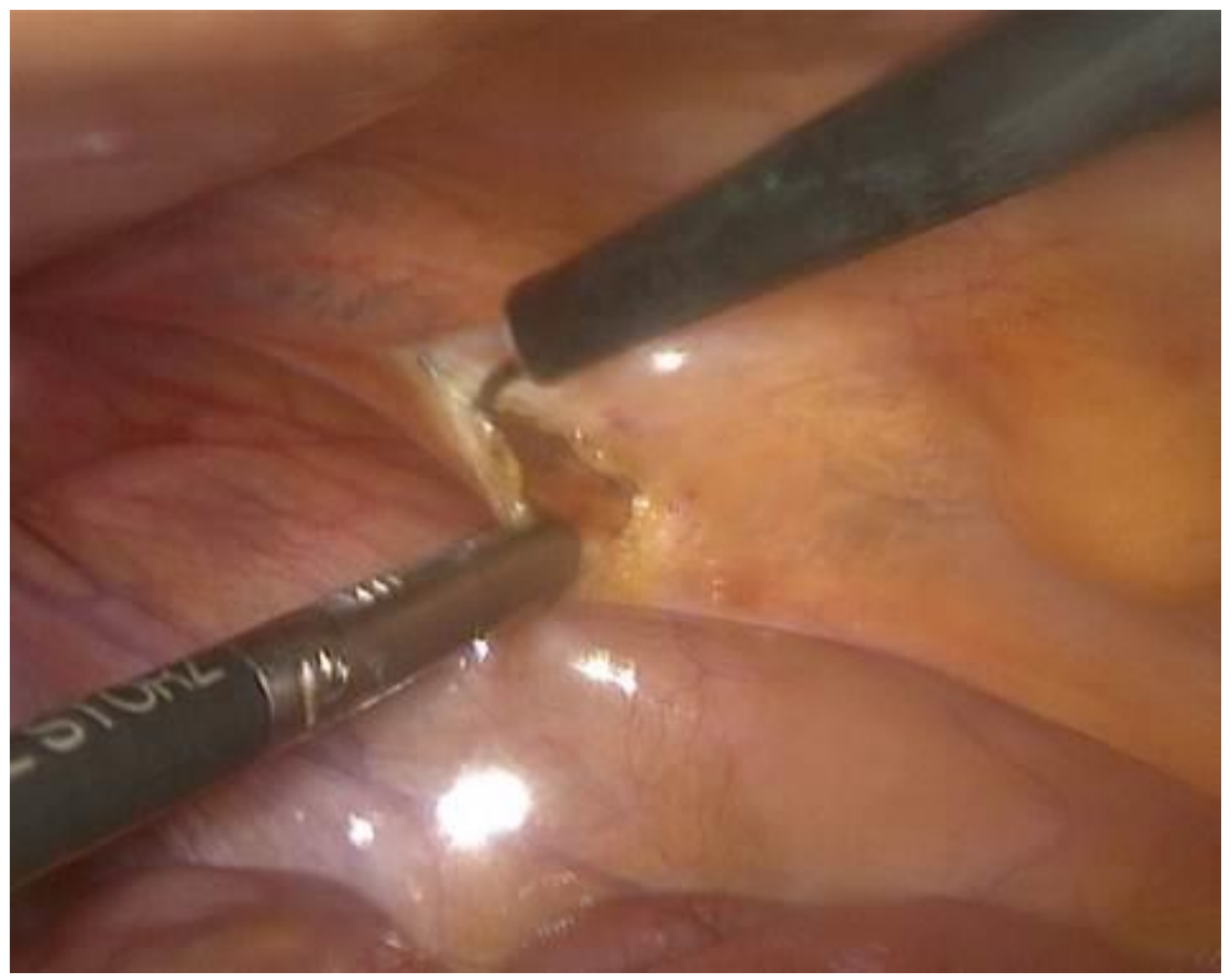

Fig. (2): Laparoscopic view of elevation of peritoneal flaps. 
https://ejhm.journals.ekb.eg/

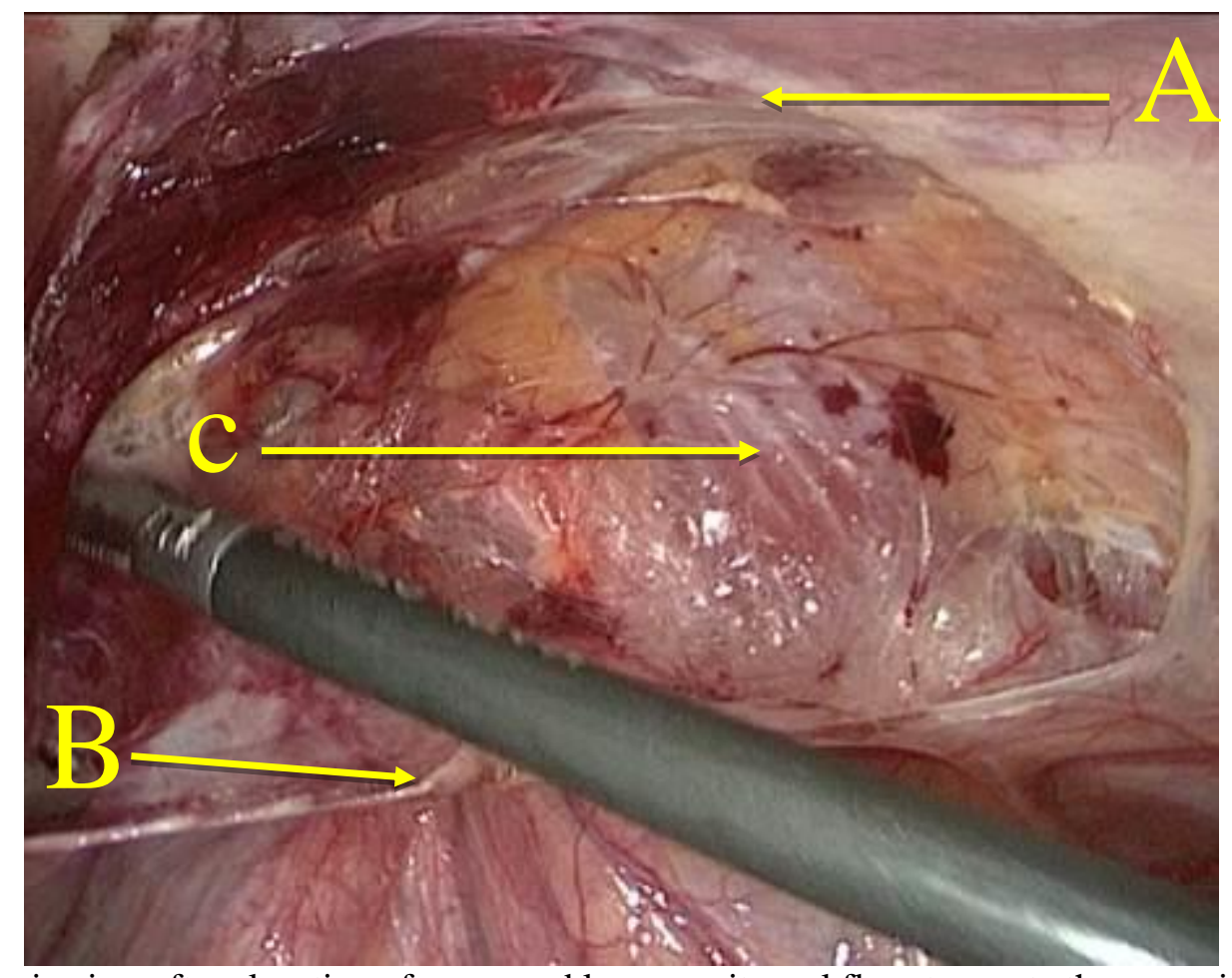

Fig. (3): Laparoscopic view after elevation of upper and lower peritoneal flaps to create the preperitoneal space. (A: the upper peritoneal flap, B: the lower peritoneal flap and C: the preperitoneal space).

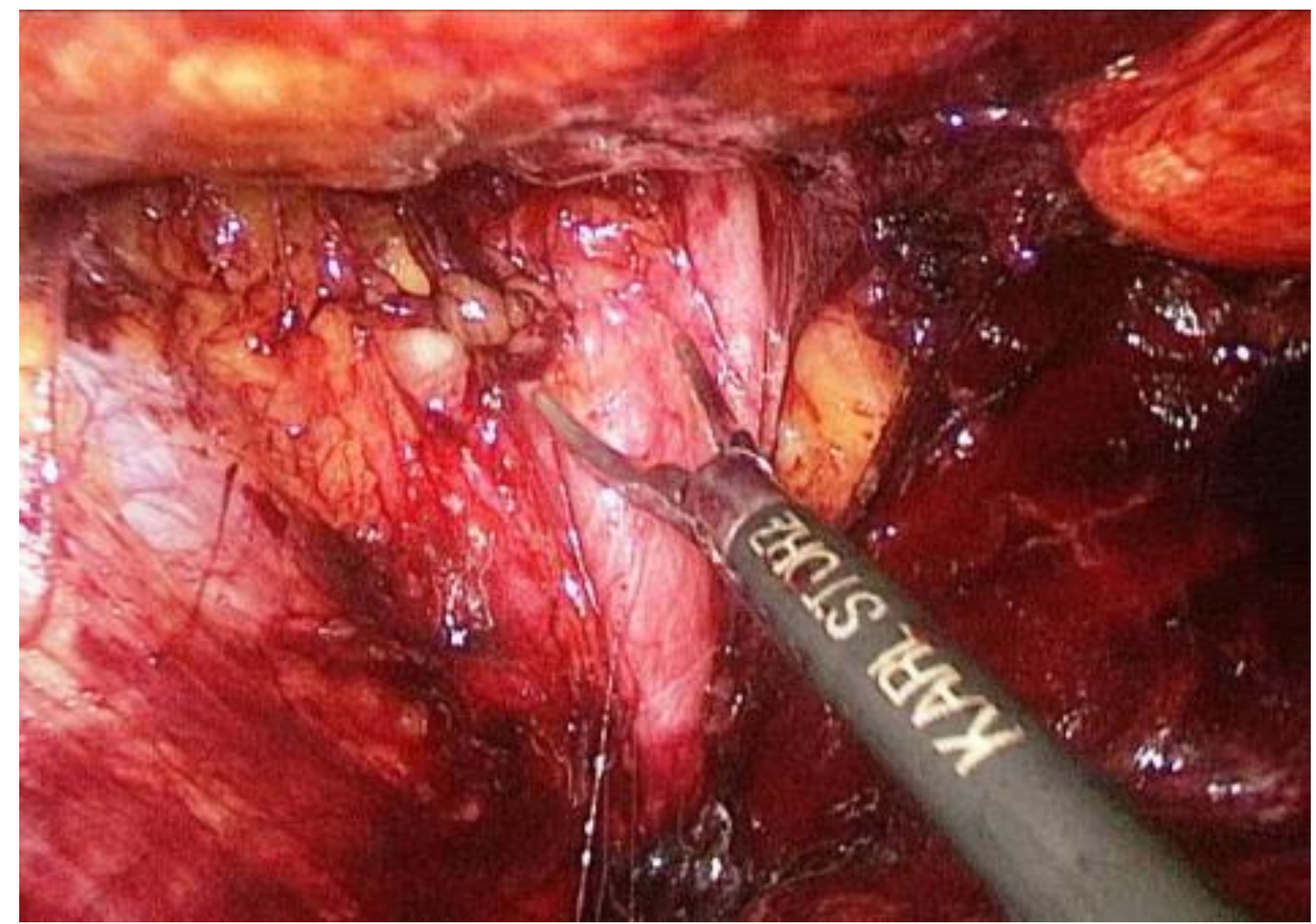

Fig. (4): Dissection of the sac from the other constituents of the cord. 


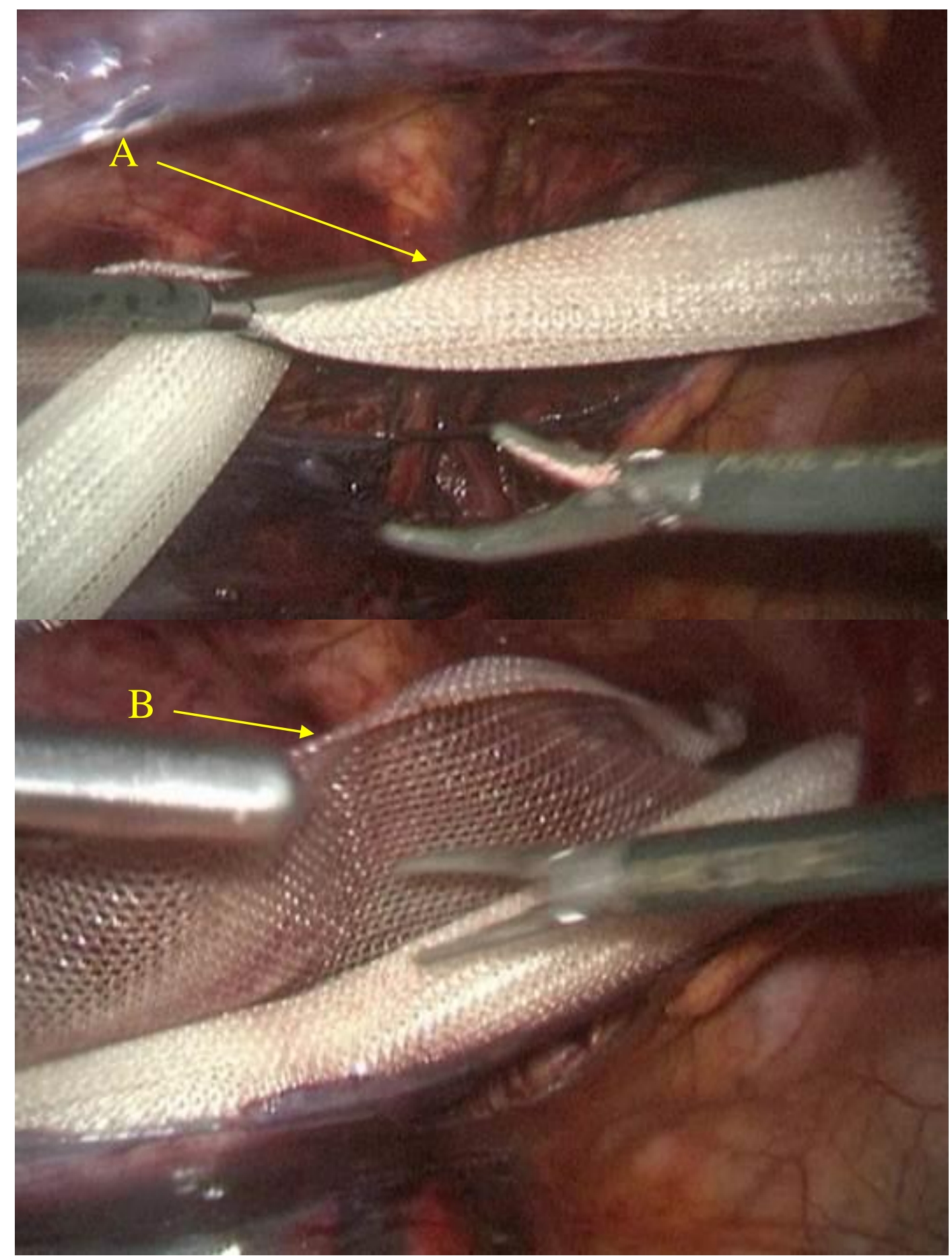

Fig. (5): Laparoscopic view of manipulation of the mesh. (A: showing the enrolled mesh, B: showing the beginning of mesh manipulation by two graspers to flatten it before fixation). 
https://ejhm.journals.ekb.eg/

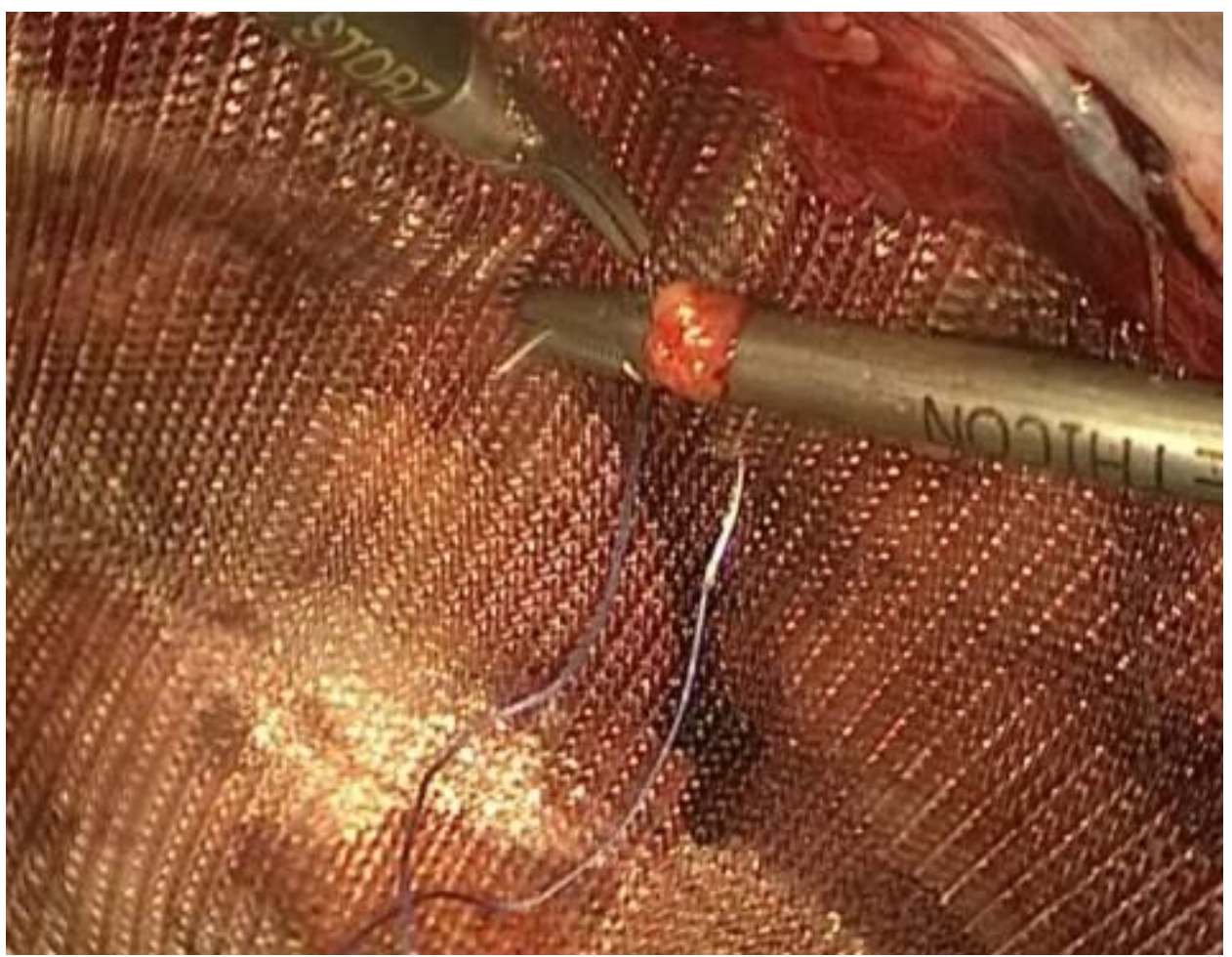

Fig. (6): Laparoscopic view of fixation of the mesh in the preperitoneal space.

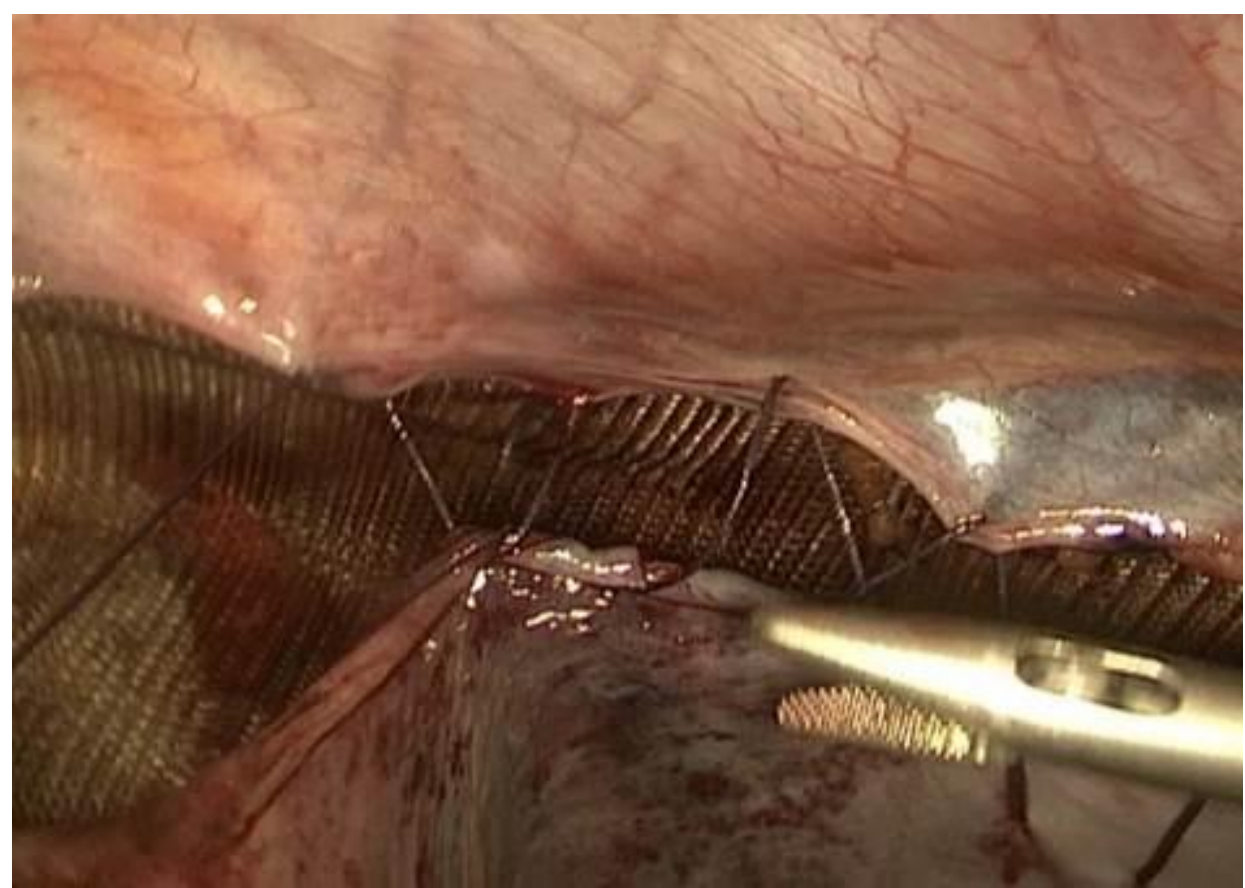

Fig. (7): Laparoscopic view of closure of the peritoneal flaps.

\section{Follow-up:}

Patients were followed-up in the outpatient's clinic for recurrence, capability of work, and rapidity to return to work, capability to perform daily activities, follow-up was scheduled on postoperative day 7 , and then every two weeks during the first month postoperatively, and then once monthly during the next six months.

\section{Statistical analysis}

The collected data were coded, processed and analyzed using the SPSS (Statistical Package for the
Social Sciences) version 22 for Windows ${ }^{\circledR}$ (IBM SPSS Inc, Chicago, IL, USA). Qualitative data were represented as frequencies and relative percentages. Quantitative data were expressed as mean \pm SD (Standard deviation) and median.

\section{RESULTS}

This study included 50 patients experienced laparoscopic inguinal hernia repair for primary inguinal hernia. Patients' demographics are listed in table 1 . 
Table (1): Patients' demographics and preoperative data

\begin{tabular}{|c|c|c|}
\hline Variable & Number of hernias & Percentages \\
\hline \multicolumn{3}{|l|}{ Age in years } \\
\hline - 20-30 & 30 & $60 \%$ \\
\hline - $\quad 31-40$ & 12 & $24 \%$ \\
\hline - $41-50$ & 6 & $12 \%$ \\
\hline - 51-60 & 2 & $4 \%$ \\
\hline \multicolumn{3}{|l|}{ Gender } \\
\hline - Male & 45 & $90 \%$ \\
\hline - Female & 5 & $10 \%$ \\
\hline \multicolumn{3}{|l|}{ Occupation } \\
\hline - Workers & 30 & $60 \%$ \\
\hline - Employee & 10 & $20 \%$ \\
\hline - Students & 5 & $10 \%$ \\
\hline - Housewives & 3 & $6 \%$ \\
\hline - Non workers & 2 & $4 \%$ \\
\hline \multicolumn{3}{|l|}{ Previous surgery } \\
\hline - Cholecystectomy & 3 & $6 \%$ \\
\hline - Cesarean section & 2 & $4 \%$ \\
\hline - Appendectomy & 2 & $4 \%$ \\
\hline - No previous surgery & 43 & $86 \%$ \\
\hline Body mass index & $24.12 \pm 4.378$ & \\
\hline \multicolumn{3}{|l|}{ Type of hernia } \\
\hline - Unilateral indirect & 29 & $58 \%$ \\
\hline $\begin{array}{l}\text { - Bilateral mixed direct and } \\
\text { indirect }\end{array}$ & 13 & $26 \%$ \\
\hline - Unilateral direct & 6 & $12 \%$ \\
\hline - Occult combined & 2 & $4 \%$ \\
\hline \multicolumn{3}{|l|}{ Precipitating factors } \\
\hline - Chronic cough & 4 & $8 \%$ \\
\hline - Heavy workers & 8 & $16 \%$ \\
\hline - Smokers & 12 & $24 \%$ \\
\hline - No definite factor & 26 & $52 \%$ \\
\hline
\end{tabular}

Regarding the operative outcomes; the operative times were longer for bilateral hernias than unilateral hernias and quite longer in oblique hernias than direct hernias. The operative time for the first 10 patients was long but it decreased after that. In respect of the intraoperative complications; accidental injury of inferior epigastric artery occurred in one patient $(2 \%)$ and controlled with clips, serosal tears of small bowel occurred in two patients (4\%), which were repaired laparoscopically with Vicryl $^{\circledR}$ 3.0. Overall, the excessive dissection resulted in minimal blood loss from the spermatic fascia and extraperitoneal fat so the amount of blood loss was scanty (Table 2).

Regarding postoperative course, oral feeding started after 6 hours after surgery for all patients and 40 patients (80\%) were ambulant and practicing all activities after being fully conscious. Patients had an uneventful postoperative recovery period and were discharged the next day on routine oral analgesics (diclofenac $50 \mathrm{mg}$ plus paracetamol 50 $\mathrm{mg}$ ) twice daily for days as required.

In respect to postoperative wound pain during the first 24 hours, 10 patients (20\%) suffered from persistent pain in the form of numbness and burning pain in the inguinal region and treated with non-steroidal anti-inflammatory drugs. The nerve irritation resolved in two months after surgery. Early postoperative complications, was encountered in the form of surgical emphysema that occurred in three patients (6\%) but was self-limited and resolved spontaneously. Postoperative hospital stay ranged from 24-48 hours with a mean of $26.04 \pm 4.13$ hours (Median 24). Our patients returned to full activities in $7.14 \pm 0.96$ days and returned to work in $14 \pm 2.5$ days. Fortunately, no mortality, no persistent pain, no recurrence, and the mean time to return to full activities was $7.14 \pm 0.96$ days (Table 2). 
Table (2): Operative and postoperative outcomes

\begin{tabular}{|c|c|c|}
\hline Variable & $\begin{array}{l}\text { Number } \\
\text { of hernias }\end{array}$ & Percentages \\
\hline $\begin{array}{c}\text { Operative time } \\
\text { (Mean time in minutes } \\
\pm \text { SD) } \\
-\quad 1^{\text {st }} 10 \text { operations } \\
-\quad 2^{\text {nd }} 15 \text { operations } \\
-\quad 3^{\text {rd } 25 \text { operations }}\end{array}$ & \multicolumn{2}{|c|}{$\begin{array}{c}(125 \pm 12.5) \\
(96.9 \pm 25.8) \\
(69.8 \pm 24)\end{array}$} \\
\hline Vascular injury & 1 & $2 \%$ \\
\hline Visceral injury & 2 & $4 \%$ \\
\hline Amount of blood loss & \multicolumn{2}{|c|}{ Scanty } \\
\hline $\begin{aligned} & \text { Postoperative pain } \\
& \begin{array}{l}\text { - } \\
\text { - }\end{array} \text { Mild } \\
& \text { - } \text { Pedersistent } \\
& \text { moderate } \\
&\end{aligned}$ & $\begin{array}{c}35 \\
5 \\
10\end{array}$ & $\begin{array}{l}70 \% \\
10 \% \\
20 \%\end{array}$ \\
\hline $\begin{array}{l}\text { Hospital Stay in hours } \\
\text { (Median 24) } \\
\text { - } \quad 24-30 \\
\text { - } 31-36 \\
\text { - } 37-48\end{array}$ & $\begin{array}{c}38 \\
9 \\
3\end{array}$ & $\begin{array}{c}76 \% \\
18 \% \\
6 \%\end{array}$ \\
\hline Surgical emphysema & 3 & $6 \%$ \\
\hline Wound infection & 0 & $0 \%$ \\
\hline Small bowel obstruction & 0 & $0 \%$ \\
\hline Scrotal hematoma & 0 & $0 \%$ \\
\hline $\begin{array}{l}\text { Persistent postoperative } \\
\text { pain }\end{array}$ & 0 & $0 \%$ \\
\hline Return to full activities & \multicolumn{2}{|c|}{$7.14 \pm 0.96$ days } \\
\hline Return to work & \multicolumn{2}{|c|}{$14 \pm 2.5$ days } \\
\hline Recurrence & 0 & $0 \%$ \\
\hline
\end{tabular}

\section{DISCUSSION}

Although inguinal hernia repair is one of the most common elective general surgical operations, there is no universal agreement on the ideal repair. In the beginning of the 1990s, laparoscopic hernia repair was controversial because of the reported high recurrence rates reaching $25 \%$ of patients ${ }^{(15)}$. But, after a decade of experience in laparoscopic hernia repair surgery, it had made significant strides becoming the first choice for inguinal hernia repair in different centers ${ }^{(16)}$. Laparoscopic repair has got wide acceptance as the gold standard of dealing with bilateral and recurrent inguinal hernias. However, open hernia repair remains commonly performed. Unlike operations such as cholecystectomy, the laparoscopic technique has not superseded open operations in terms of numbers being done ${ }^{(4)}$. Surgeons tend to perform open surgery for patients who had open primary inguinal hernia surgery and they even did not consider the recommendation of laparoscopic surgery for recurrent hernia ${ }^{(17)}$.

Although one of the disadvantages of TAPP is the prolonged operative time ${ }^{(\mathbf{1 8})}$, yet there is no significant difference in operation time between the mesh-plug, Lichtenstein, TAPP, and totally extra-peritoneal (TEP) techniques ${ }^{(\mathbf{1 9})}$. In Krishna et al. (20) study, the mean operative time was $72.3 \pm 25.9 \mathrm{~min}($ range $=30-130 \mathrm{~min})$. In our study, the operative time ranged between (60-130) minutes with a mean time $(81.2 \pm 22.12)$ minute. We have slight increase in the mean operative time which started longer at the beginning $(125 \pm 12.5)$ then became shorter with experience (69.8 24$)$ because of the steep learning curve.

Laparoscopic approach allows viewing the myopectineal orifice and repairing any unexpected hernia thereby reducing the chance of recurrence $^{(21)}$. Such occult hernias are often overlooked during open repair and may require re-operation, especially if located on the contralateral side. With laparoscopic inguinal hernia repair, these occult hernias can be easily detected and repaired during the same secession without additional incisions ${ }^{(22)}$. Sayad et al. ${ }^{(23)}$ and Koehler ${ }^{(24)}$ have previously reported the rate of occult contralateral hernias found during TEP repair is 11 and $13 \%$, respectively. In our study occult combined hernias were detected in $4 \%$ of patients where there were a combination of direct inguinal hernia and femoral hernia detected during laparoscopic repair and accordingly patients avoided re-operation, exposure to second anesthesia, and another period of work loss, which is advantageous.

Studies have reported an intraoperative bowel injury rate of $0-0.06 \%$ in laparoscopic hernia repair ${ }^{(25)}$. The injury can be repaired intraoperatively if the surgeon is experienced ${ }^{(26)}$. In our study, visceral injury occurred in 2 patients (4\%) and was serosal tears that was stitched laparoscopically. Actually, we have high percentage of visceral injury but we owe this to the early experience of this method and also these injuries occurred in the early start of the study and with experience we didn't report visceral injury.

Vascular injury is a relatively uncommon but nonetheless catastrophic complication. During preperitoneal dissection, the inferior epigastric vessels sometimes become separated from the abdominal wall and then hang down into the operative field, division of these vessels between clips may be needed to complete the procedure ${ }^{(27,28)}$. In Krishna et al. (20) study, there were no major vascular or inferior epigastric vessel injuries. In our study, accidental injury of inferior epigastric artery occurred in one patient (2\%) and was controlled with clips.

Small bowel obstructions have been reported after laparoscopic repair due to herniation of bowel loop through the port site or through a defect in peritoneal closure ${ }^{(29,30)}$. Kapiris et $\boldsymbol{a l}^{\left({ }^{(31)}\right.}$ in their study had $(0.23 \%)$ cases of small bowel obstruction due to herniation 
through a peritoneal gap. At the beginning of their study, they used staples for peritoneal closure, but later they used sutures to close the peritoneal gap after mesh fixation. They noticed decreased incidence of this complication following sutured peritoneal closure. In ours we did not experience any patient had bowel obstruction as we used continuous running suture using Vicryl ${ }^{\circledR}$ 2-0 without any gap to prevent adhesions of bowel or omentum and herniation through any defect.

Postoperative pain is the most common complaint after hernia surgery. There is great advantage for laparoscopy over open methods is less postoperative pain, thus reducing the incidence of chronic pain ${ }^{(29)}$. In Krishna et al. ${ }^{(20)}$ study, the overall pain score for laparoscopy was not significantly high. In our study, $70 \%$ of the patients suffered from mild pain and took single dose of injected analgesic, $10 \%$ of the patients suffered from moderate pain, which responded to double dose of injected analgesic, $20 \%$ of the patients suffered from moderate pain but persistent pain, the patients took the non-steroidal anti-inflammatory drugs for two weeks and then the pain resolved. Our policy is to anchor the mesh above the iliopubic tract and this avoids nerve injury erroneously ${ }^{(27)}$, also the repair with flat, low weight mesh reduces pain ${ }^{(28)}$.

Recurrence is the most important outcome of any hernia surgery ${ }^{(32)}$. The key to minimize the recurrence rate is thorough knowledge of anatomy and a thorough technique of repair ${ }^{(33)}$. The complete wide exposure and coverage of the entire myopectineal orifice ${ }^{(34)}$ through the use a large-sized piece of mesh; the mesh must be large enough to extend $2 \mathrm{~cm}$ medial to the pubic tubercle, $3-4 \mathrm{~cm}$ above the Hesselbach triangle, and $5-6 \mathrm{~cm}$ lateral to the internal ring ${ }^{(26)}$. In Krishna et al. ${ }^{(20)}$ study, they did not notice any recurrence in the immediate postoperative period and during follow-up ranging from 15 to 38 months (average $=29.5$ months). In our study there was no evidence of recurrence till the end of the study and the postoperative follow up period, and we consider this point as very important.

The laparoscopic repair is more expensive than open repair but the major advantages which include postoperative pain reduction, shorter hospital stay and earlier return to work can decrease the $\operatorname{cost}^{(35)}$. In our patients the postoperative hospital stay ranged from 24-48 hours with a mean of $26.04 \pm 4.13$ hours, they returned to full activities in $7.14 \pm 0.96$ days and returned to work in $14 \pm 2.5$ days.

\section{CONCLUSION}

TAPP repair is a feasible and safe technique, resulting in less postoperative pain associated with rapid recovery, early mobilization and discharge from the hospital, as well as early return to usual activities and less postoperative complication. In the same time it allows detection and repair of occult contralateral defects. It is recommended as the procedure of choice in elective groin hernia repair especially in bilateral cases.

\section{REFERENCES}

1. Shrestha B, Shrestha S (2020): Analysis of acute postoperative pain in patients undergoing transabdominal preperitoneal (TAPP) repair of inguinal hernia in Manipal Teaching Hospital. Nepal Journal of Medical Sciences, 5:71-78.

2. Scott N, Go P, Graham P et al. (2001): Open mesh versus non-mesh for groin hernia repair. Cochrane Colorectal Cancer Group (ed). Cochrane Database Syst Rev., 68:259-65.

3. Fitzgibbons R, Forse R (2015): Clinical practice. Groin hernias in adults. N Engl J Med., 372:75663.

4. Trevisonno M, Kaneva $P$, Watanabe $Y$ et al. (2015): Current practices of laparoscopic inguinal hernia repair: a population-based analysis. Hernia, 19:725-33.

5. Ielpo B, Duran H, Diaz E et al. (2018): A prospective randomized study comparing laparoscopic transabdominal preperitoneal (TAPP) versus Lichtenstein repair for bilateral inguinal hernias. Am J Surg., 216:78-83.

6. Avtan L, Kasik L, Onarımi F (2011): Transabdominal preperitoneal (TAPP), hernioplasty. Laparosc Endosc Surg Sci., 18:1227.

7. Feleshtynskyi Y, Shtaier A (2020): Morphological justification of laparoscopic transabdominal preperitoneal (TAPP) operation for the inguinal hernia recurrence. Journal of Education, Health and Sport, 10:455-63.

8. Hernia Surge Group (2018): International guidelines for groin hernia management. Hernia, 22:1-165.

9. Barbaro A, Kanhere H, Bessell J et al. (2017): Laparoscopic extraperitoneal repair versus open inguinal hernia repair: 20-year follow-up of a randomized controlled trial. Hernia, 21:723-27.

10. Neumayer L, Giobbie-Hurder A, Jonasson $O$ et al. (2004): Open mesh versus laparoscopic mesh repair of inguinal hernia. N Engl J Med., 29:181927.

11. Hallén M, Bergenfelz A, Westerdahl J (2008): Laparoscopic extraperitoneal inguinal hernia repair versus open mesh repair: long-term followup of a randomized controlled trial. Surgery, 143:313-7.

12. Kukleta J (2006): Causes of recurrence in laparoscopic inguinal hernia repair. J Minim Access Surg., 2:187-91.

13. Zogg C, Pawlik T, Haider A (2018): Three common methodological issues in studies of 
surgical readmission rates. JAMA Surg., 153:1074-6.

14. Ranjani C, Paniraj A (2020): A study of abdominal wall hernias and its management. International Journal of Scientific Study, 8:73-6.

15. Fitzgibbons R, Camps J, Cornet D (1995): Laparoscopic inguinal herrnioraphy: results of a multicenter trail. Ann Surg., 221:3-13.

16. Dulucq J, Wintringer $P$, Mahajna A (2008): Laparoscopic totally extraperitoneal inguinal hernia repair: lessons learned from 3,100 hernia repairs over 15 years. Surg Endosc., 23:482-6.

17. Lee C, Kim J, Choi B et al. (2020): Retrospective study on prevalence of recurrent inguinal hernia: a large-scale multi-institutional study. Ann Surg Treat Res., 98:51-55.

18. Yasukawa D, Aisu Y, Hori T (2020): Crucial anatomy and technical cues for laparoscopic transabdominal preperitoneal repair: Advanced manipulation for groin hernias in adults. World $\mathrm{J}$ Gastrointest Surg., 12:307-25.

19. Shi Y, Xiao D, Dai L et al. (2020): Comparison of the effect of mesh-plug, Lichtenstein, transabdominal preperitoneal, and totally extraperitoneal hernia repair: A network metaanalysis. Rev Assoc Med Bras., 66:687-91.

20. Krishna A, Misra M, Bansal V et al. (2012): Laparoscopic inguinal hernia repair: transabdominal preperitoneal (TAPP) versus totally extraperitoneal (TEP) approach: a prospective randomized controlled trial. Surg Endosc., 26: 639-49.

21. Barrat C, Surlin V, Bordia A et al. (2003): Management of recurrent inguinal hernias: a prospective study of 163 cases. Hernia, 7:125-9.

22. van den Heuvel B, Munoz Brands $R$, Beuerle $E$ et al. (2015): A rare case of a groin hernia: the Hessel-bach's hernia. Hernia, 19:523-6.

23. Sayad $P$, Abdo $Z$, Cacchione $R$ et al. (2000): Incidence of incipient contralateral hernia during laparoscopic hernia repair. Surg Endosc., 4:543-5.

24. Koehler $\mathbf{R}$ (2002): Diagnosing the occult contralateral inguinal hernia. Surg Endosc., 16: 512-20.
25. Lau H, Patil N, Yuen $W$ et al. (2003): Prevalence and severity of chronic groin pain after endoscopic totally extraperitoneal inguinal hernioplasty. Surg Endosc., 17:1620-3.

26. Shaiban M, Khushaim L, Alghamdi $\mathbf{R}$ et al. (2018): Laparoscopic versus open inguinal hernia repair in adults. EJHM., 70: 392-6.

27. Sajid M, Kalra L, Parampalli U et al. (2013): A systematic review and meta-analysis evaluating the effectiveness of lightweight mesh against heavyweight mesh in influencing the incidence of chronic groin pain following laparoscopic inguinal hernia repair. Am J Surg., 205:726-36.

28. Bringman S, Blomqvist $P$ (2005): Intestinal obstruction after inguinal and femoral hernia repair: a study of 33,275 operations during 1992 2000 in Sweden. Hernia, 9:178-83.

29. Dalessandri K, Bhoyrul S, Mulvihill S (2001): Laparoscopic hernia repair and bladder injury. JSLS., 5:175-7.

30. Kingsnorth A, LeBlanc $K$ (2003): Hernias: Inguinal and incisional. Lancet, 362:1561-71.

31. Kapiris S, Brough W, Royston C et al. (2001): Laparoscopic transabdominal preperitoneal (TAPP) hernia repair: A 7-year two- center experience in 3017 patients. Surg Endosc., 15:972-5.

32. Bringman S, Ek A, Haglind E et al. (2001): Is a dissection balloon beneficial in totally extraperitoneal endoscopic hernioplasty (TEP)? A randomized prospective multicenter study. Surg Endosc., 15:266-70.

33. Knook M, Weidema W, Stassen L (1999): Endoscopic total extraperitoneal repair of bilateral inguinal hernias. Br J Surg., 86:1312-6.

34. Fitzgibbons R, Puri V (2006): Laparoscopic inguinal hernia repair. Am Surg., 72:197-206.

35. Shimizu S, Uchiyama A, Mizumoto $\mathrm{K}$ et al. (2000): Laparoscopically assisted distal gastrectomy for early gastric cancer. Is it superior to open surgery? Surg Endosc., 14:27-31. 